Proceedings of XIX International Scientific Conference "New Technologies and Achievements in Metallurgy, Material Engineering, Production Engineering and Physics", Częstochowa, Poland, June 7-8, 2018

\title{
The Effectiveness of Eye Protection Application against Harmful Blue Radiation in Modern Mobile Devices
}

\author{
M. SROGA ${ }^{a}$, M. DośPiAe ${ }^{a, *}$ AND M. GACEK ${ }^{a, b}$ \\ ${ }^{a}$ Faculty of Production Engineering and Materials Technology, Institute of Physics, \\ Częstochowa University of Technology, al. Armii Krajowej 19, 42-200 Częstochowa, Poland \\ ${ }^{b}$ Silesian Center for the Treatment of Eye Diseases, Dworcowa 8, 44-240 Żory, Poland
}

\begin{abstract}
This study compares the quality of eye protection against the blue electromagnetic radiation, in the range of a filters, programmed in the various types of mobile devices. In order to carry out a comparison, the emission spectrum of displays were compared with each other, before and after starting off the filter. The received data were collated with the spectral efficiency curves for thermal and photochemical hazard. Additionally, there was made an analysis of changes in the quality of color mapping, by using the device's programmed protection filters.
\end{abstract}

DOI: 10.12693/APhysPolA.135.162

PACS/topics: vision: light detection, adaptation, and discrimination; color vision: color detection, adaptation, and discrimination, photometry

\section{Introduction}

Technological progress significantly accelerates by giving us, day by day, more amenities such as televisions, computers, and smartphones. On the streets, standard billboards are replaced with screens that can display images which change one after another, or even movies, and conventional lighting on the streets or in houses is replaced by LED lamps. Thanks to the use of LED technology (light-emitting diode), you can build much smaller and cheaper displays, and lighting devices that also consume significantly less electricity. Unfortunately, it is at the expense of our health, due to the introduction of environmental changes in the field of ocular photometry $[1,2]$. White LEDs used to illuminate displays of our electronic devices largely emit harmful, bioactive blue radiation [3].

Nowadays almost everyone has a smartphone and uses it for many hours through a day, exposing their eyes to the complications associated with excessive exposure to blue light such as insomnia, photochemical damage, earlier occurrence of cataracts, and AMD [4-6]. In order to save the user from harmful radiation at least in part, program filters are used.

The subject of this work is to determine to what extent the available software filters protect the vision organ from excessive exposure to blue radiation and how they affect the color reproduction of the displayed image.

\section{Research methodology, material for testing}

In the research, a portable Al-Prof SM-H1 spectrometer was used, which enables photometric testing in all conditions. The basic function of the apparatus is the

\footnotetext{
*corresponding author; e-mail: mdospial@wp.pl
}

ability to measure the emission spectrum of any radiation source in the range from 320 to $810 \mathrm{~nm}$. In addition, the device collects data on light intensity, color temperature, gamut and CRI, or color coefficient.

The research material consisted of various models of commercially available smartphones, in which the manufacturer used a software filter that limits blue light emissions.

In order to standardize the results of measurements, the phone displays were always brightened to the maximum allowed by the software, and the measurement was carried out on a white background. The spectrometer head, in order to extinguish the same measurement conditions, was always applied directly to the screen at right angles. The smartphones were not protected by polycarbonate foils.

\section{Research results and their discussion}

Figure 1 shows the emission spectrum from the phone display Huawei P9 Lite 2017 (red line). It can be distinguished by three broad emission peaks having the maximum distribution values for the wavelength $\lambda$ equal to $452,540,615 \mathrm{~nm}$, and with half widths $21,51,55 \mathrm{~nm}$. The peak in short wavelengths corresponds to the blue radiation range and has the highest intensity. Its maximum is almost exactly in the range marked in the literature as the most bioactive for which the relative spectral efficiency of $B_{\lambda}$ causing visible radiation of photochemical damage of the retina is 0.97 [1]. This indicates a significant bioactivity of the matrix on the photoreceptors of the eye containing melanopsin. After using the eye protection function (curve marked in red) in the form of a program filter, the intensity of the blue radiation peak decreases (about 57\% intensity), while maintaining the same radiation pattern in the medium and long wave spectrum. This is better seen in Fig. 2, which shows the difference between the two spectra. 


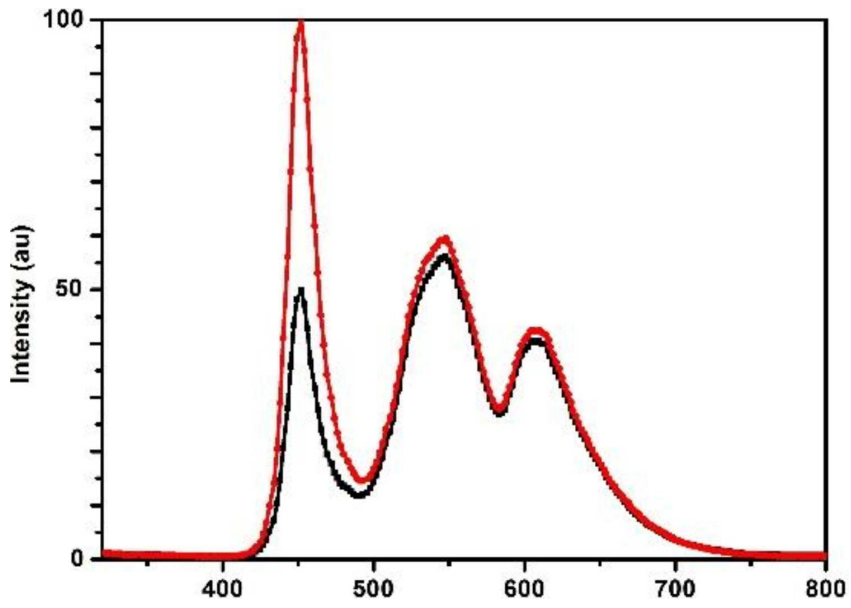

Fig. 1. Emission spectrum with no protection (red) and with protection (black).

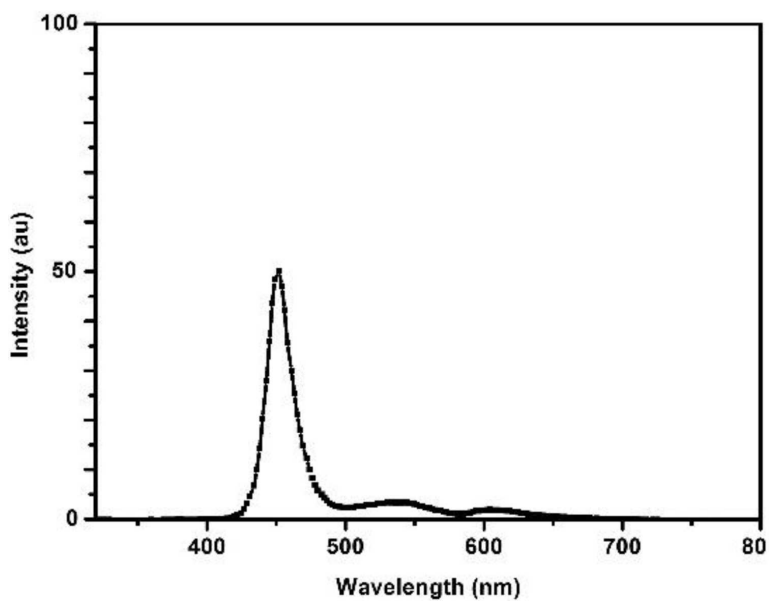

Fig. 2. Difference between the spectrum with and without protection.

On the basis of both graphs, it can be concluded that software protection accomplishes its tasks by reducing the intensity of the blue peak by more than half. It should be noted that this is the CRI parameter of the entire screen, which is an indicator of the color reproduction calibrated to sunlight (or a blackbody), where 100 describes the perfect reproduction. If a software filter is used, it drops from 91.5 to 61.5 , which is shown in Fig. 3. The color of the screen from position $x=0.312$ and $y=0.322$, i.e. with shifted point, achromatic toward the blue green/greenish blue in the CIE diagram, after applying the filter, it shifted toward the yellowgreen/greenish-yellow color to the position $x=0.364$ and $y=0.411$. The total shift vector in the CIE chromaticity diagram had an offset value in the directions $x=0.052$ and $y=0.090$, respectively, and a resultant equal to 0.104 .

In the case of the next device, which was a Huawei smartphone P9 Lite 2016 model (Fig. 4), can be seen the emission spectrum in comparison to the previous one. The intensity in the range of yellow and red in relation

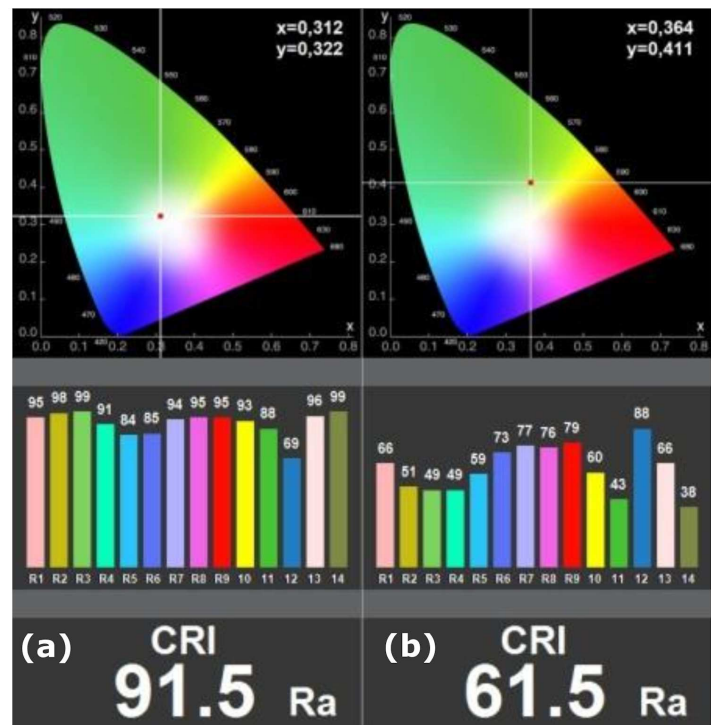

Fig. 3. Color reproduction of probes, CRI and color gamut of the tested screen (a) without protection, (b) with software protection.

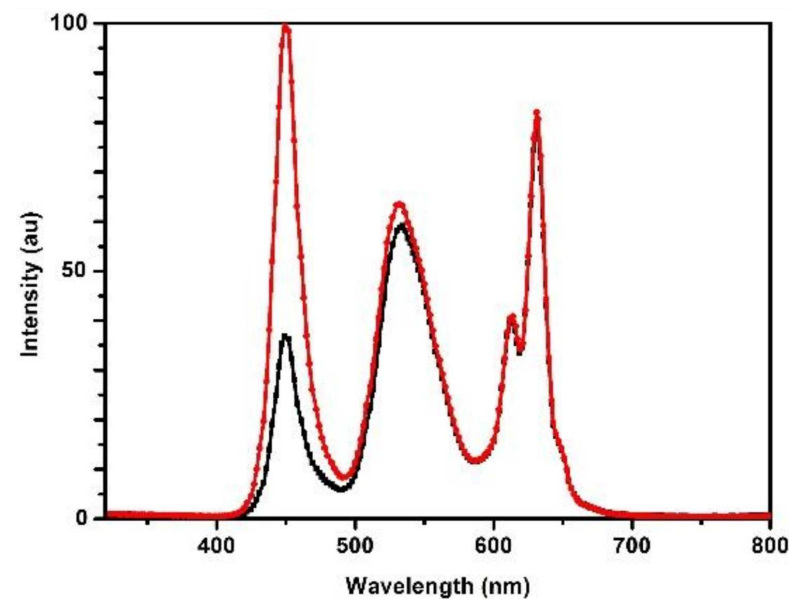

Fig. 4. Emission spectrum with no protection (red) and with protection (black).

to the blue has significantly increased, which indicates the use of a different display. As before, three broad emission peaks can be distinguished having the maximum distribution values for the wavelength $\lambda$ equal to 450, 536, $627 \mathrm{~mm}$ and half widths, respectively 20, 45, $27 \mathrm{~nm}$. The peak for the blue range, as in the previous case, has the highest intensity, but this time its intensity only slightly dominates over the other components of the spectrum. Its maximum, as before, coincides with the maximum bioactivity of blue radiation $B_{\lambda}=0.94$. This allows us to state that the matrix studied emits much less blue light, but still in a strongly bioactive spectrum area. After using the eye protection function, the intensity of the blue radiation peak decreases by approximately $63 \%$, and the intensity of peaks in the medium and long wave spectrum is also slightly weakened. The difference in radiation emission is shown in Fig. 5. 


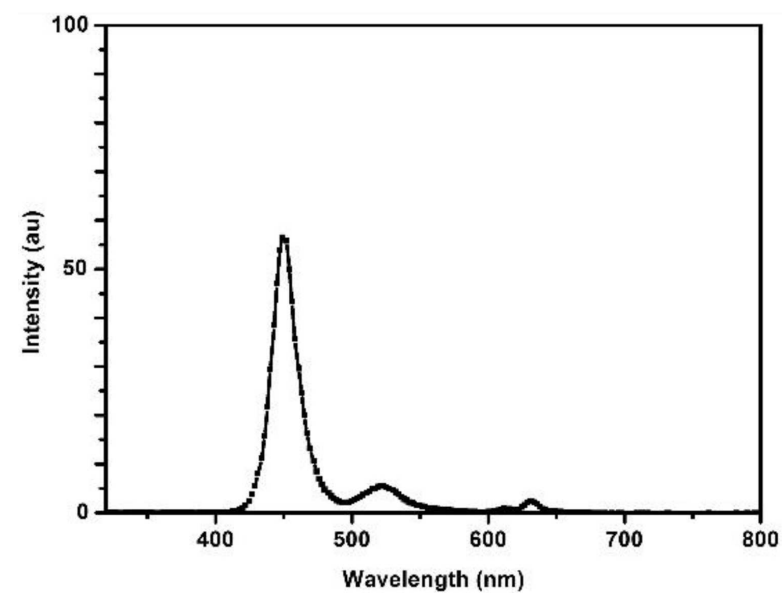

Fig. 5. The difference between the spectrum with and without protection.

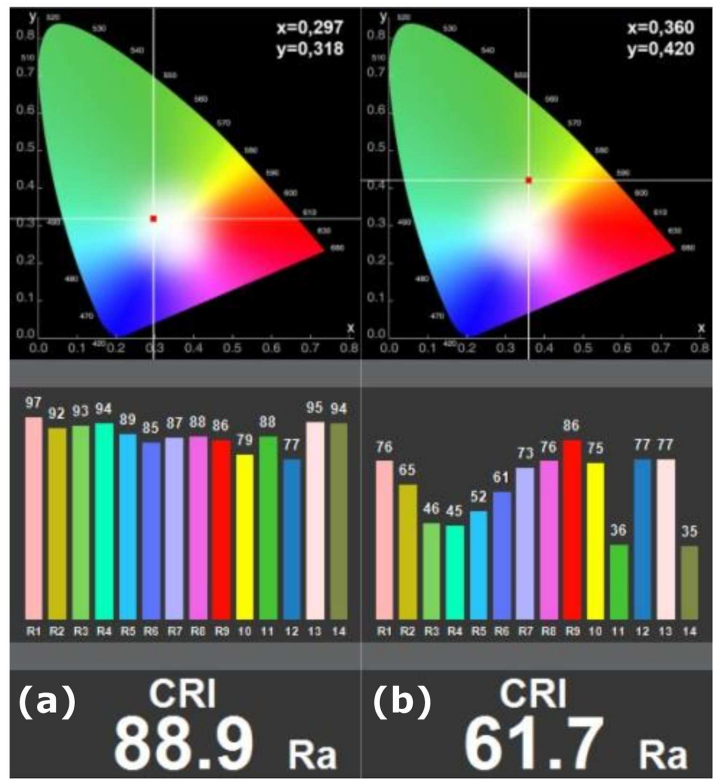

Fig. 6. Color reproduction of probes, CRI and color gamut of the tested screen (a) without protection, (b) with software protection.

As with the previous filter, the eye protection function is also used.

In the case of the matrix described, the CRI color mapping quality parameter was initially lower (88.9) and the achromatic point of the matrix was shifted towards blue green/greenish blue to position $x=0.297$ and $y=0.318$. The use of a software filter shifts the achromatic point of the matrix to the position $x=0.360$ and $y=0.420$ in the yellow-green/greenish-yellow direction and significantly reduces the quality of CRI mapping to 61.7, as illustrated in Fig. 6. The total shift vector in the $x$ and $y$ directions is 0.063 and 0.102 . However, its total value is 0.120 .

The last emission chart of the display belongs to the smartphone Samsung S8 films. The spectrum is dominated by three peaks with intense intensity: the color of

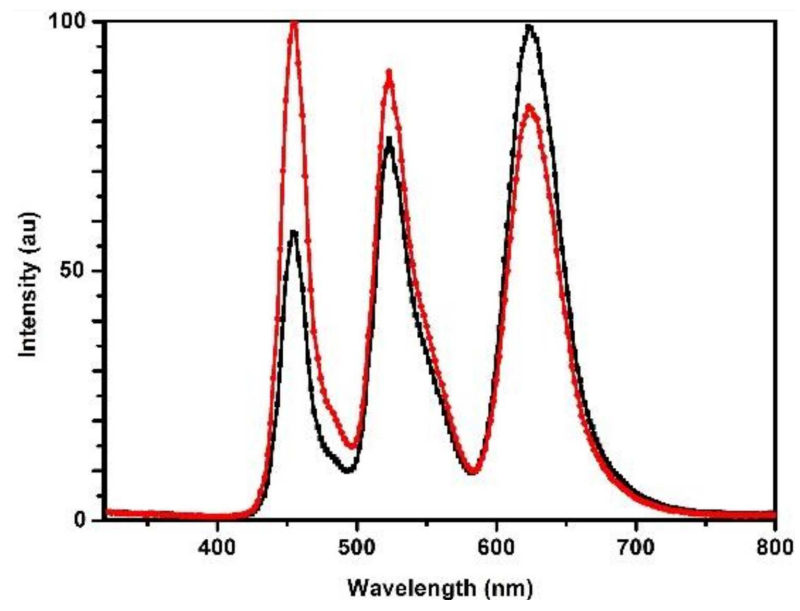

Fig. 7. Emission spectrum with no protection (red) and with protection (black).

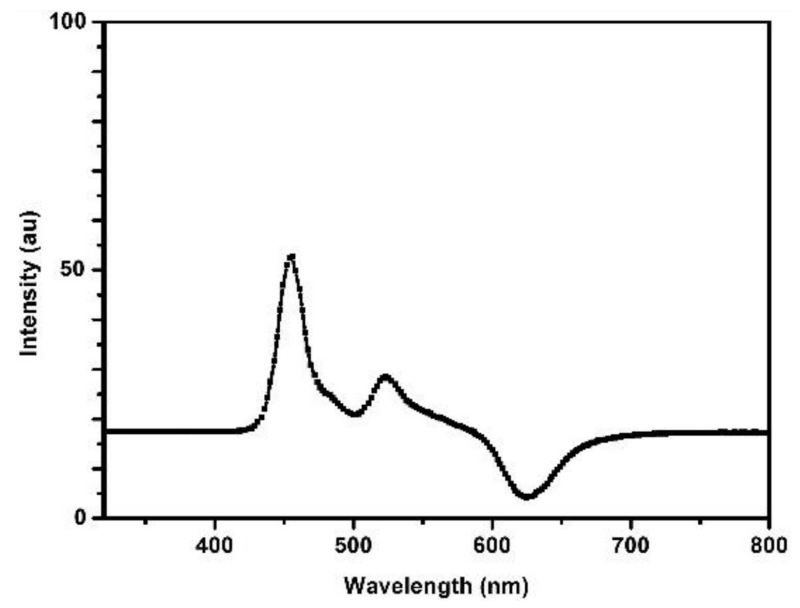

Fig. 8. The difference between the spectrum with and without protection.

the sky near $455 \mathrm{~nm}$, yellow at $520 \mathrm{~nm}$ and red at $630 \mathrm{~nm}$. It definitely distinguishes it from previously tested phone screens. The position of the maximum peak for the blue color lies on the arm of the function of the distribution of the bioactivity of blue radiation and its value $B_{\lambda}$ is equal to 0.90 . After applying the program filter, the intensity of the blue peak decreases, however by the lowest value by $35 \%$, also the intensity for the yellow peak decreases slightly, and the peak for the red light increases as shown in Fig. 7. Figure 8 shows the differences between the emission spectrum without protection (red graph), and spectrum with software protection (black graph). In comparison to the previous differential plots, there is a marked increase in the intensity of the red color.

On the basis of the presented graphs 7 and 8, it can be concluded that the matrix of the third phone is characterized by the lowest bioactivity, but the programmable eye protection function correlated with this display works the worst.

In the case of the third matrix, the achromatic point almost coincided with the values of $x=1 / 3, y=1 / 3$ 


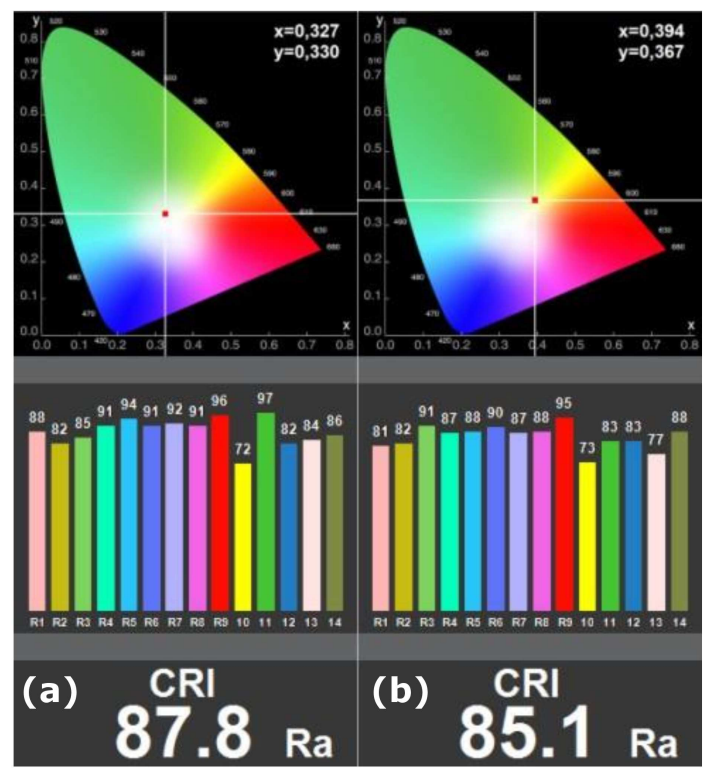

Fig. 9. Color reproduction of probes, CRI and color gamut of the tested screen (a) without protection, (b) with software protection.

and had the position $x=0.327$ and $y=0.330$ (deviation respectively for the $x$ and $y$ axis, 0.006 and 0.003$)$. The use of a software filter shifted the achromatic point of the matrix to the position $x=0.394$ and $y=0.367$ in the yellow/yellowish-orange direction and only slightly reduced the quality of CRI mapping from 87.8 to 85.1 , as illustrated in Fig. 9. Total vector shift in $x$ and $y$ directions is 0.067 and 0.037 , respectively. However, its total value is 0.076 .

\section{Conclusions}

Based on the collected results, it can be concluded that all telephones had a strong blue component in the radiation spectrum. In addition, the positions of peaks for blue light correlated with the location of the maximum distribution of retinal bioactivity in the context of photochemical damage to the eye. The weakest intensity of the blue component was recorded in the third matrix, where, moreover, the shift of the bioactivity index on its distribution was noted, which in this case was 0.9 (compared to 0.97 and 0.94 in other matrices).

Software protection used in mobile devices significantly reduces the emission of blue light. The only exception was the last of the examined eyesight protection functions (acting on the third sensor), which significantly reduces the radiation in this range by only $35 \%$ (relative to the first and second matrix, respectively 57 and $63 \%$ ).
In the case of the first two matrices, the achromatic point was shifted towards the blue-green/greenish-blue color which indicates the abuse of the backlight with blue light. The third of the matrices seems optimized in this context because its achromatic point assumes values practically equal to $1 / 3$ for both $x$ and $y$ coordinates. Optimization of the achromatic position in this case increased safety for the visual system of the matrix used.

Filters used to reduce the amount of blue light in mobile devices noticeably affected the reproduction of colors by the screen and the resultant gamut of displays. The change in the scope of white mapping significantly changes not only the color impression but also brings a significant change in the contrast between background and text. This raises further questions regarding the changes that eye protection filters cause in the context of e.g. the cognitive abilities of processed texts [7] with reduced mutual contrast in the function of the background color. The above studies may provide important boundary data in the conduct of such research.

\section{References}

[1] Handbook of Occupational Safety and Health, Ed. D. Koradecka, CRC Press, Boca Raton 2010.

[2] PN-EN 14255-2:2010 "Pomiar i ocena ekspozycji osób na niespójne promieniowanie optyczne - Część 2: Promieniowanie widzialne i podczerwone emitowane przez źródła sztuczne na stanowisku pracy" (in Polish).

[3] J.K. Sheu, S.J. Chang, C.H. Kuo, Y.K. Su, L.W. Wu, Y.C. Lin, W.C. Lai, J.M. Tsai, G.C. Chi, R.K. Wu, IEEE Photon. Technol. Lett. 15, 18 (2003).

[4] Shen-Shen Yan, Wei Wang, Int. J. Ophtalmol. 9, 1066 (2016).

[5] E.A. Boettner, J. Reimer Wolter, Invest. Ophthalmol. Vis. Sci. 1, 776 (1962).

[6] A.R. Wielgus, R.J. Collier, E. Martin, F.B. Lih, K.B. Tomer, C.F. Chignella, J.E. Roberts, Photochem. Photobiol. Sci. 9, 1505 (2010).

[7] N. Bonnardel, A. Piolat, L. Le Bigot, Displays 32, 69 (2011). 